Bull. Mater. Sci., Vol. 4, No. 2, April 1982, pp. 109-123. (C) Printed in India.

\title{
Convection phenomena at reduced gravity of importance in space processing of materials
}

\author{
SIMON OSTRACH \\ Case Western Reserve University, Cleveland, Ohio 44106, USA
}

MS recejved 19 June 1981

\begin{abstract}
The basic aspects of convection processes are delineated. It is shown that even in weak gravitational fields buoyancy can induce fluid motions. Furthermore, at reduced-gravity other non-gravity forces such as surface or interfacial tensions, $g$-jitter, thermal-volume expansions, density differences due to phase changes, and magnetic and electric fields can induce fluid motions.

The various types of flow possible with these various driving forces are described and criteria for determining the extent and nature of the resulting flows and heat transfer are presented. The various physical mechanisms that can occur separately and in combination are indicated and the present state of knowledge of each of the phenomena is outlined.
\end{abstract}

Keywords. Reduced-gravity convection; thermal convection; solutal convection ; thermosolutal convection ; surface-tension convection ; thermoacoustic convection ; phase-change convection; coupled convection.

\section{Introduction}

It is a common impression that in the reduced gravity environment, say $10^{-6} \mathrm{~g}$, of an orbiting spacecraft, convection cannot occur and hence such manufacturing processes where convection is inevitable in $1-g$ environment of earth, is deleterious and can be performed more successfully. Thus, much enthusiasm has been expressed for utilizing spacecraft for such processes. However, initial experiments carried out in Apollo, Skylab and other spacecraft have indicated, to the contrary, that convection is not absent (Grodzka and Bannister 1972; Bannister 1973), and indeed can be present in significant amounts. The basic reason for this is that in the space environment there are a variety of non-gravity forces that can induce fluid motion, which can be further influenced by the reduced $g$-levels encountered in spacecraft (tables 1 and 2). Such non-gravity driving forces which are usually suppressed by gravity include surface or interfacial tensions, thermal volume expansions, magnetic and electric fields and finally $g$-jitter, caused by onboard vibrations from machinery or astronaut movements, attitude control manoeuvres, etc.

Due to all the above complexities it has become necessary for scientists planning materials processing experiments in space to know the extent and nature of convection in space and factors controlling it. This information could be used to minimize the effects of convection or to utilize it to advantage. A rather comprehensive presentation of the various types of natural convection possible in space 
manufacturing processes is given by Grodzka (1973). Related existing publications are also indicated therein. In Grodzka and Bannister (1974) some of the convection types are related to space-flight experiments. The present article outlines key aspects related to natural convection of various types and its effects in reduced gravity environment.

\section{Nature of convection}

Most configurations related to materials processing are ones in which the fluid is confined by rigid boundaries. Internal convection problems are considerably more complex than external ones because the fluid boundary layer and core are closely coupled (Ostrach 1972). This constitutes the main source of difficulty in predicting the resulting flow and heat transfer. More than one core configuration (flow) is sometimes possible and which will actually occur for a given set of conditions cannot be determined a priori..... Furthermore, the entire flow is sensitive to the configuration geometry and to the imposed thermal boundary conditions.

To add to the complexity there are essentially two basic modes of flow generated by a body force (Ostrach 1964). The first, which will be referred to as conventional convection, oxcurs whenever a density gradient (due to thermal, concentration, or other effects) is normal to the body force (gravitational) vector. In sucb a case flow results immediately. The second mode, which will be called unstable convection, results whenever the density gradient is parallel but opposed to the body force. In this situation the fluid remains in a state of unstable equilibrium (due to heavier fluid being above lighter fluid), until a critical density gradient is exceeded. A spontaneous flow then ensues which ratber quickly becomes steady. This motion is usually in the form of cells or vortex rolls and, hence, causes more mixing than laminar conventional convection. It must be emphasized that this instability is different from the one that results in laminar flow becoming turbulent. The steady flow obtained after the critical condition is exceeded is laminar; this flow, (as all others dut to buoyancy), can also become turbulent under proper conditions. The onset of the unstable motion is highly dependent on the confining boundaries, i.e. the motion can be markedly delayed by proper design of the configuration (Ostrach and Pnueli 1963 ; Sherman and Ostrach 1967).

As if all the above were not sufficiently difficult to deal with it should be pointed out that both modes of convection, conventiona! and unstable, can also occur in a given configuration (for example, see Hart 1971, 1973).

As was indicated above, the space environment furtber complicates the picture because of the additional driving forces. Further complexities arise in materials processing because the fluid is not bomogeneous so that concentration gradients can cause density gradients in addition to temperature gradients. The density gradients due to those two causes can enbance or oppose each other in coupled ways. Relatively little work exists for normal natural convection witb multiple coupled driving gradients and, particularly, for the types of geometric configurations that correspond to materials processing methods. It is thus, perbaps not surprising that the effects and nature of natural convection under reduced-gravity conditions are often neglected, misinterpreted, or confused. 
From the above discussion it should be clear that, because different modes of convection are possible and they are extremely sensitive to the configuration and boundary conditions, in order to assess convection effects at reduced gravity the following information must be specifically and explicitly known:

(i) Both the maguitude and direction of accelerations; the latter to help determine the mode of convection,

(ii) The geometric configuration,

(iii) The imposed boundary conditions,

(iv) The material properties.

Unfortunately, most of the existing literature on this subject is deficient in one or more of these.

\section{General considerations}

For reasons given above it is necessary to study each prot lem by itself to obtain detailed information on the transport processes. However, considerable insight into the qualitative nature of the problem can be obtained from dimensional analysis. The first step in the understanding of such a complex phenomenon, therefore, is to obtain the relevant dimensionless parameters. These are best determined from the basic equations and toundary conditions that describe the phenomena (Ostrach 1966).

Parameters - For convection generated by an acceleration acting on a density difference (or gradient) the parameters are:

(a) The Grashof number, Gr, which represents the ratio of buoyancy to viscous forces

$$
\mathrm{Gr}=g \frac{\Delta \rho d^{3}}{\rho v^{2}},
$$

where $\Delta \rho$ denotes the density difference, $g$ the acceleration, $d$ a characteristic dimension, $\rho$ a reference density, and $v$ the kinematic viscosity. If the density difference is due to a temperature difference (or gradient) (1) becomes

$$
\mathrm{Gr}=\frac{\beta g \triangle T d^{3}}{v^{2}}=\frac{\beta g(\partial T / \partial x) d^{4}}{v^{2}}
$$

where $\beta$ is the fluid volumetric coefficient, $\Delta T$ is the temperature difference, and $(\partial T / \partial x)$ denotes a spatial temperature gradient. Note that for gases $\beta=1 / T$ where $T$ is the absolute reference temperature.

(b) The Rayleigh number

$$
\mathrm{Ra}=\left(c_{\mathrm{p}} \mu / k\right) \mathrm{Gr}=\operatorname{PrGr},
$$

where $c_{p}$ is the specific heat at constant pressure, $\mu$ is the absolute viscosity, $k$ the thermal conductivity coefficient, and $\mathrm{Pr}$ is the Prandtl number and is a measure of the ratio of the diffusion of vorticity to heat.

The Grash of number basically appears in the equations of motion and, therefore, the fluid velocities are directly related to it. For large Grasbof numbers $(\mathrm{Gr}>1)$ the fluid velocities, $U$ can be estimated from

$$
U \pm \sqrt{\mathrm{Gr}} \frac{v}{d}=(\beta g \triangle T d)^{\frac{1}{2}},
$$


whereas for small Grashof numbers $(\mathrm{Gr}<1)$

$$
U=\mathrm{Gr} \frac{\nu}{d}=\frac{\beta g \triangle T d^{2}}{v} .
$$

The Rayleigh number, on the other hand, appears in the energy equation so that the heat transfer is related to it.

If the density difference in (1) is due to a difference in concentration, then (1) becomes

$$
\mathrm{Gr}_{\mathrm{s}}=a g \Delta c d^{3} / v^{2}=\alpha g(\partial c / \partial x) d^{4} / v^{2}
$$

where $\alpha$ is the concentration densification coefficient, $\Delta c$ is the concentration difference, and $\partial c / \partial x$ represents a spatial concentration gradient. The corresponding solutal Rayleigh number is

$$
\mathrm{Ra}_{\mathbf{1}}=\alpha g(\partial c / \partial x) d^{4} / v D
$$

where $D$ is the diffusion coefficient.

Since fluid motions are of primary concern in most materials processing problems, the procedure to abtain an estimate $\mathrm{cf}$ the velocities is as follows: From the direction of acceleration and the configuration determine which made of convection is to be expected. For conventional convection compute the Grashof

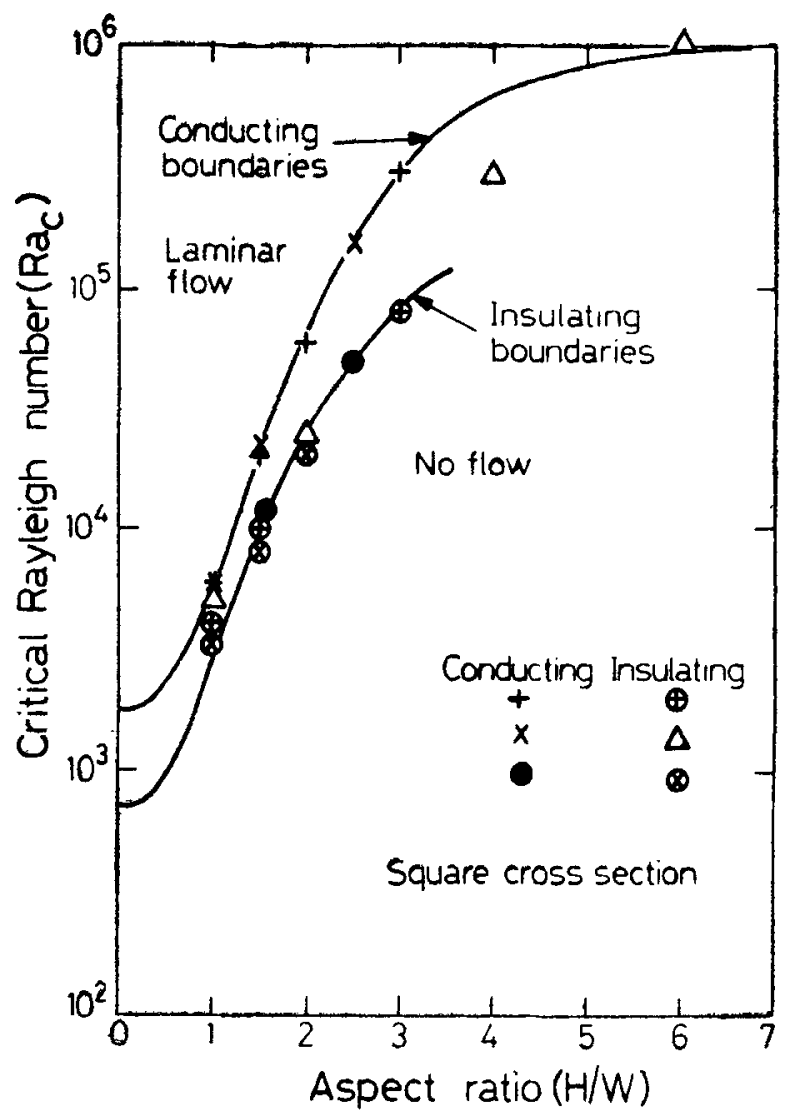

Figure 1. Effect of aspect ratio on critical Rayleigh number for vertical cylinders. 
number from the appropriate equation (1), then use the corresponding velocity expression (3). If unstable convection is anticipated, the proper critical Rayleigh number for the configuration must be determined to see if such a flow will actually occur. In addition to Ostrach and Pnueli 1963; Sherman and Ostrach 1967 there is an increasing number of papers in which critical Rayleigh numbers are determined for various configurations subject to different conditions. Space limitations preclude a detailed discussion of them (Ostrach 1976). However, the essential physical aspects of the problems can be illustrated by figures 1 and 2. The effect of aspect ratio on the critical Rayleigh number is shown in figure 1 for vertical cylinders of square cross-section. with both conducting and insulated vertical boundaries. The solid lines represent theoretical results of Samuels and Churchill (1967), Davis (1967), Catton (1970) and Jennings and Sani (1972) and the experimental data came from Cattor, and Edwards (1967), Heitz and Westwater (1971) and Edwards and Catton (1969). There is good agreement between theory and experiment regardless of whether the cross-section is rectangular or circular, although the form of the resulting flows may be different. Increasing the aspect ratio (containment) car be seer. to be very stabilizing. The critical Rayleigh number is, however, insensitive to the aspect ratio for values of it greater than 5 .

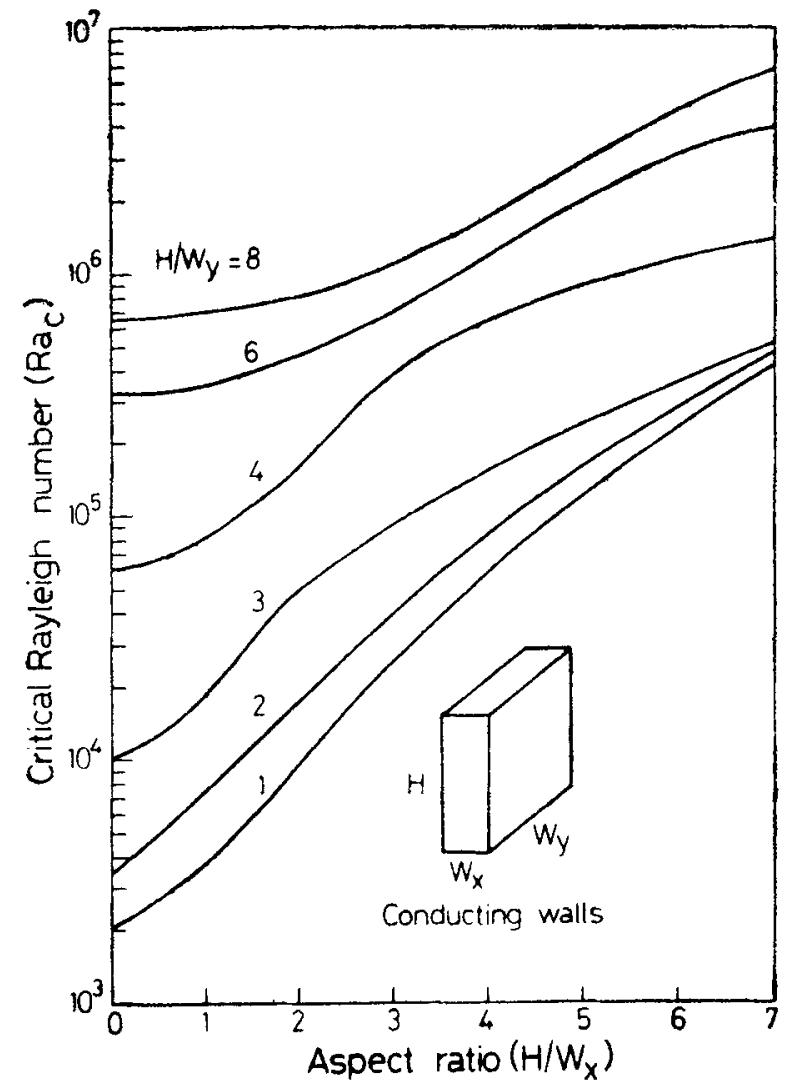

Figure 2. Critical Rayleigh number as a function of aspect ratios. 
The stability of fluids in rectangular parallelepipeds heated from below was theoretically determined by Catton (1972) for a wide range of thermal boundary conditions on the lateral walls. Some of the results are presented in figure 2 where the critical Rayleigh number is plotted against as pect ratios for various rectangular cross-sections. It is evident that with increasing cross-sectional confinement (large $\left.\boldsymbol{H} / W_{y}\right)$ that the configuration is even more stable than for square or circular cross-sections for which $H / W_{s}$ and $H / W_{y}$ are equal. This indicates another design option for delaying the onset of unstable convection.

When a rectangular cavity with one wall hotter than the other is rotated with respect to the gravity vector, or vice versa, each of the natural convection modes, conventional and unstable, is obtained as limits and for angles in between both modes interact. The types of flow are delineated (Hart 1971, 1972) and a summary of the results is shown in figure 3 . The Rayleigh number is based on the distance and the temperature difference between the hot and cold walls of the container. When the hot surface is below $\left(\delta=90^{\circ}\right)$ there is no convection until the classical Benard cells appear at $\mathrm{Ra}_{0}=1708$. At $\delta=-90^{\circ}$ the hot surface is on top and the fluid layer is stably stratified and again there will be no convection. However, when the slot is nearly vertical or rotated so that the hot wall is above, a steady circulating two-dimensional unicellular flow occurs with fluid rising along the hot surface and descending near the cold surface. Longitudinal vortex rolls develop when the two basic modes interact or when the Rayleigh number is sufficiently large and wavy flow patterns result.

Other aspects of the combined modes are treated by Unny (1972), Hollands and Konicek (1973) and Clever (1973) for very shallow boxes. The combined effects of aspect ratio and inclination angle on the circulation and heat transfer rate in a
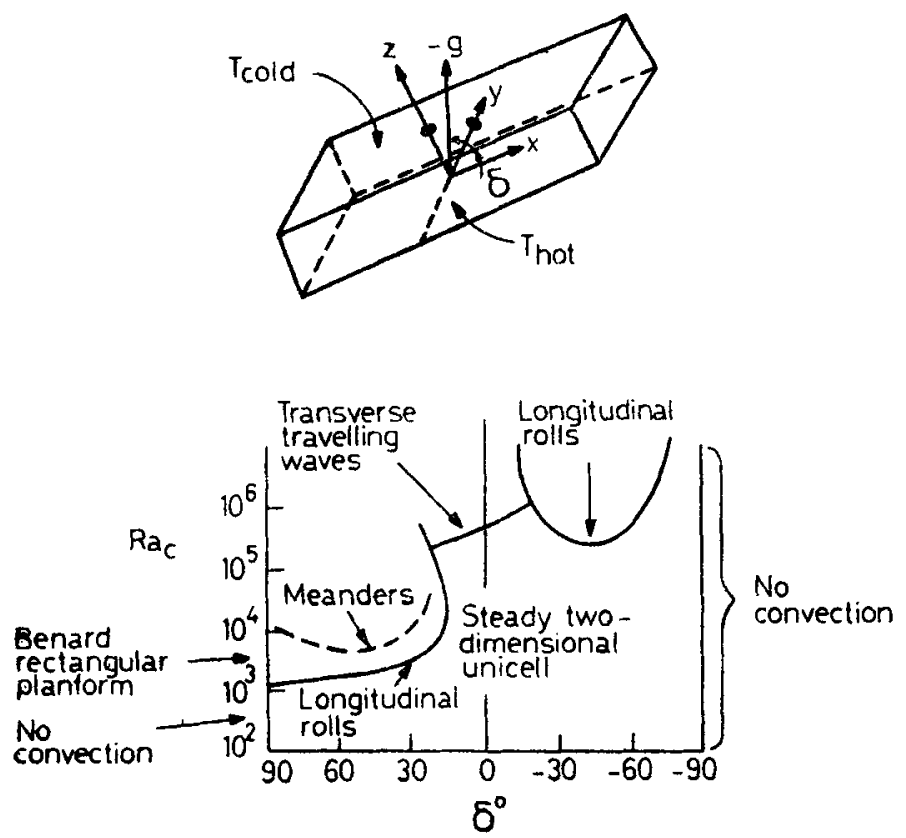

Figure 3. Flow types with combined modes of convection. 
finite rectangular region were investigated primarily theoretically by Ozoe et al $(1974 a, b)$ and experimentally by Ozoe et al (1975). It was found that when the angle of inclination from horizontal was zero (unstable configuration) the flow pattern was a series of roll cells with axes parallel to each other and perpendicular to the long axes of the channel. When the inclination angle was increased slightly in steps a series of roll cells persisted with their axes in the upslope but the average Nusselt number (beat transfer) decreased. As the angle was increased further a minimum Nusselt number was attained. Beyond that angle the flow pattern changed to a single roll cell with its axes in the long dimension of the channel and the associated Nusselt number increased. This flow pattern persisted as the angle was increased until the heated surface was horizontal but above the cold one. The Nusselt number passed through a maximum and then decreased to unity. This behaviour was qualitatively the same for channels with aspect ratios from unity to fifteen but the inclination angles corresponding to the Nusselt number extrema depend strongly on the aspect ratio. Comparis on of the theoretical and experimental results indicated that the results of Ozoe (1974b) can be utilized up to $\mathbf{R a}=10^{5}$ and aspect ratios up to at least $8 \cdot 4$.

Scaling - The dimensionless parameters are extremely useful for reasons in addition to that of obtaining flow estimates. They indicate how to scale a problem, for example, to reduce convection or to study the phenomena in a model. They also indicate possible simplifications for analysis, the minimum number of experiments necessary, and how best to correlate data (see Ostrach 1966).

\section{Buoyancy-induced convection}

The acceleration due to gravity on earth is essentially constant at a value of $980 \mathrm{~cm} / \mathrm{sec}^{3}$ (one-g). A steady and reduced value of acceleration is inherent in most spacecraft because of atmospheric drag, centripetal force due to vehicle rotation, gravity gradients, solar wind, and solar pressure. Superposed on this background of a uniform but weak acceleration field are temporarily-varying accelerations that are due to engine burns, attitude-control manoeuvres, and onboard vibrations from machinery of astronaut movements.

Steady Micro-Gravity Fields-Some of the steady accelerations associated with several missions are summarized in tables 1 and 2 (taken from Chin et al 1969 and Forester 1971 and presented by Grodzka and Bannister 1974).

From the values of the accelerations presented above it would appear that the $g-$ levels are small and, therefore, that the environment is one of zero gravity and no convection is possible. This is the misconception that is so prevalent in much of the space processing work. However, the basis for estimating both thermal and solutal convection effects was given above. Under the most mundane condition, e.g., temperature differences of $10^{\circ} \mathrm{K}$ at levels of about $20^{\circ} \mathrm{C}$, and a characteristic length of $10 \mathrm{~cm}$ the Grashof number (for thermal convection) at one-g (equation 1 (a)) for a gas (like air) is of the order of $10^{6}$, for a liquid (water) is like $10^{7}$, and for a liquid metal (mercury) is about $10^{9}$. Thus, it is clear that even in reduced-gravity levels as indicated in the tables the Grashof number is not negligible. For example, at $10^{-5} \mathrm{~g}$ velocities of the order of tenths of a millimeter per second would be induced in the liquid and liquid 
Table 1. Summary of stcady reduced-grdvity accelorations.

\begin{tabular}{llll}
\hline \multicolumn{1}{c}{ Source } & \multicolumn{3}{c}{ Type of mission } \\
\cline { 2 - 4 } & $\begin{array}{c}\text { Low-altitude earth } \\
\text { orbit }\end{array}$ & $\begin{array}{c}\text { Low altitude } \\
\text { lunar orbit }\end{array}$ & $\begin{array}{c}\text { Interplanetary } \\
\text { trajectory }\end{array}$ \\
\hline Atmospheric drag & $5 \times 10^{-5} \mathrm{~g}$ and lower & None & None \\
Centripetal force & $1 \times 10^{-6} \mathrm{~g}$ & $3 \times 10^{-7} \mathrm{~g}$ & $3 \times 10^{-14} \mathrm{~g}$ \\
Gravity gradient & $3 \times 10^{-9} \mathrm{~g} / \mathrm{cm}$ & $2 \times 10^{-9} \mathrm{~g} / \mathrm{cm}$ & $4 \times 10^{-17} \mathrm{~g} / \mathrm{cm}$ \\
\cline { 2 - 4 } Venting thrust & All $10^{-4} \mathrm{~g}$ to $10^{-6} \mathrm{~g}$, neglecting drag \\
Vehicle thrust & All $0.1 \mathrm{~g}$ to $6 \mathrm{~g}$ & \\
\hline
\end{tabular}

Table 2. Steady g-levels in Apollo missions.

\begin{tabular}{lc}
\hline Apollo flight mode & Typical g-level \\
\hline Passive thermal control (PTC) & $3 \times 10^{-6} \mathrm{~g}$ \\
Attitude hold in translunar or transcarth orbit & $7 \times 10^{-8} \mathrm{~g}$ \\
Attitude hold in lunar orbit & $5 \times 10^{-7} \mathrm{~g}$ \\
\hline
\end{tabular}

metal under the given conditions and about four times that in the gas. Further increases in the temperature (or density) difference and length would lead to even higher fluid velocities. Similar considerations apply for solutal convection. However, since concentration gradients in materials processing can be considerably greater than temperature gradients, for a given $g$-level solutal convection effects could be significantly larger than those due to thermal convection. It is, thus, clear that the weak steady acceleration fields can lead to fluid motions. These motions occur immediately with no delay in the case of conventional convection. There may, however, be no corresponding immediate change in heat transfer associated with the flows under certain conditions. For example, for conventional convection in a rectangular enclosure it was found by Batchelor (1954) that the beat transfer will differ from that for pure conduction only if $\mathrm{Ra}>500(\mathrm{l} / \mathrm{d})$ where $l$ is the height and $d$ the width of the enclosure.

The nature of conventional convection at reduced gravity will be similar to that at normal gravity as summarized by Ostrach (1972). The essential difference is that in space because the Grashof numbers are significantly reduced the flow will be laminar whereas on earth it may be turbulent. Unstable convection will be jess likely in space because the associated lower Rayleigh numbers may be lower than the critical. 
Although there is, unfortunately, little experimental data on reduced-gravity convection corroborative evidence is available from studies made for the cryogenic gas storage tanks for the Apollo 14 and subsequent flights. Although these ground-based and space flight studies of reduced-gravity convection are specifically limited to an unusual ffuid (supercritical oxygen) and rather complex configurations (the Apollo tanks) the results are, nevertheless, of significance. For example, it was found that the natural-convection in the Apollo tanks was sufficient to obviate the need for forced convection by mixing fans (Rice 1971). Data from Apollo 14 (Fineblum et al 1971) indicates increased convection with increases in the vehicle rotation rate (up to $3 \mathrm{RPH}$ ) as would be expected.

$g$-jitter - As contrasted to the non-u niformities of the gravity field, some of which must be accepted as part of the natural environment of an orbital vehicle, there are transient or time-varying perturbations to the grevity field at a point. The unsteady variations which are referred to as $g$-jitter can arise from spacecraft manoeuvres and mechanical vibrations.

Spacecraft manoeuvres can produce $g$-jitter magnitudes of $10^{-4} \mathrm{~g}$ and higher. This effect can be overcome if the space processing is perfarmed during the drift mode. On the other hand, $g$-jitter caused by mechanical vibrations cannot necessarily be controlled at their sources. Mechanical vibrations that are transmitted to the experiment have the same effect as a time-varying gravity. They can be caused, for example, by astronaut movement, rotating and reciprocating machinery, and extra-vehicular activities. Arm and leg movements and console operations such as flipping switches are all signifioant in this respect (see Conway 1972). During Skylab experiments the $g$-jitter has been estimated to be as large as $10^{-3} \mathrm{~g}$ with the frequency spectrum of this random vibration from about one to several thousand hertz. Evidence of $g$-jitter in Apollo flights is presented by Grodzka and Bannister (1972) and Bannister et al (1973) and discussed in some detail by Gradzka and Bannister (1974).

Some indications of the effects of $g$-jitter on convection can be abtained by telated research stich as that of Richardson (1967), Pak et al (1970) and Gershuni et al (1970). It was found that vibrations can either stbstantially enhance or retard local heat transfer, significantly increase total hea.t transfer and drastically affect convection by altering the transitions from quiescent to laminar flow (critical Rayleigh number) and from laminar to turbulent flow. More detailed discussion of related work is presented by Spradley et al (1975). In the last reference specific consideration was given to models for evaluating $g$-jitter effects and they will be summarized in a later article in these praceedings.

This phenomenon merits special consideration not only because it represents a convection made that is suppressed under normal gravitational conditions and which is significant at reduced gravity but also because it is inherent in all spacecraft environments and cannot, therefore, be easily controlled.

\section{Surface-tension induced convection}

Surface tension on the free surface of a liquid may, under some conditions considerably affect liquid motion. The presence of an interface between two fluid phases can influence the motion of the fluids when either the interface has finite 
curvature or when the interfacial tension varies from point to point. In both cases, forces appear in the interfacial region that can affect or generate the fluid motion.

When the motion takes place in a gravitational field the relative importance of surface tension can be estimated from the Bond number, $\mathrm{Bo}_{0}=\rho g d^{2} / \sigma$, which indicates the ratio of gravitational to surface-tension forces, where $\sigma$ is the surface tension. From the Bond number it is clear that on earth surface tension is impartant $(\mathrm{Bo}<1)$ only in small scale configurations, i.e., where $d$ is very small. Therefore, most existing work on the effect of surface tension deals with flow in capillaries and thin films or the motion of droplets or bubbles or short wavelength water waves. In space, on the other hand, strface tension becomes a significant force whose influence on fluid motion must be assessed and understood.

Surface-tension gradients can arise from gradients in temperature or concentration. These surface-tension gradients can generate conventional convection flows or unstable cellular flows (see, for example Pearson (1958); Nield (1979); Scriven and Sternling 1974) just as gravity induces such convective flows. When the surface-tension gradients and density differences are due to temperature gradients the dimensionless parameter related to gravitational convection is the Rayleigh number, $\mathrm{Ra}=\beta g \theta d^{3} / \nu \kappa$ and the corresponding one for surface tension induced flows is the Marangoni number, $\mathrm{Ma}=\frac{(\partial \sigma / \partial T) \theta L}{\rho \nu \kappa}$ where $\beta$ is the fluid volumetric expansion coefficient, $\theta$ is a characteristic temperature difference and $\kappa$ is the thermal diffusivity. Note that the Marangoni number is independent of gravity. The relative magnitude of surface-tensian and buayancy forces is given by the ratio $\mathrm{Ma} / \mathrm{Ra}=(\partial \sigma / \partial T) /\left(\rho \beta g d^{2}\right)$. Note that this is just the reciprocal of a modified Bond number. Again, it can be seen from this ratio that surface tension becomes orders of magnitude greater (under identical conditions) than the buayancy in a reduced-gravity environment.

Conventional convection-There are many possible modes af flow and configurations of interest in which surface tension can be significant. Consideration will now be given to a situation in which conventional convection will accur. The flow and heat transfer of a fluid in an open container can be expected to change markedly as gravity is reduced. For example, in a normal gravitational environment the natural convection (due to buoyancy) may be turbulent (see figure 4a). In a somewhat reduced-gravitational field (say $10^{-3} \mathrm{~g}$ ) the gravity force might still predominate over the surface tension but the natural convection would be quite different (figure 4b), i.e., it would be laminar with a single cell or double cell. In these situations the free surface can be said to be passive with respect to the convection in the sense that the fluid motion is induced solely by buoyancy. However, as gravity is reduced further (say $10^{-6} \mathrm{~g}$ ) the surface tension becomes daminant and will generate the primary fluid motions. In such a case the free surface becomes an active factor in the convection process (figure $4 \mathrm{c}$ ).
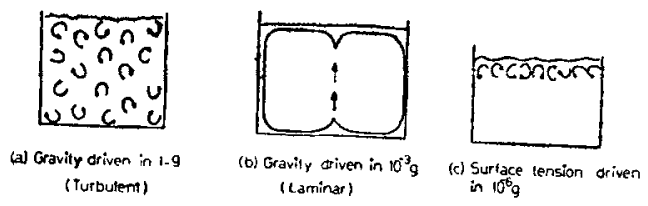

Figure 4. Modes of convection as a function of $g$-level, 
Theoretical studies of related problems are reported by Birikh (1966) and Babskiy et al (1973). An unbounded horizontal layer of fluid with a rigid lower surface and free upper surface is analysed by Birikh (1966). A linear temperature gradient is impased along both these surfaces. The velacity profiles due to buayancy alone and surface tension alone are shown in figure 5. Itcan be seen therein that near the free surface there is a flow due to surface tension from the warm region towards the cooler and a reverse flow occurs near the lower rigid surface. The profile due to buoyancy alone is of somewhat similar shape but less blunt. The flow due to surface tension in a square container with end walls at different temperatures is analyzed by Babskiy et al (1973) and the computed streamlines and isotherms are shown in figure 6. Once again the strong flow near the free surface can be seen with a reverse cell below. Several aspects of this analysis are questionable.

Since there did not appear to be any experimental study of conventional convection due to surface tension alone the present author designed such an experiment to be carried out in the NASA Lewis Research Centre's 500-foot drop tower in which approximately $5 \frac{1}{2} \mathrm{sec}$ of reduced gravity $\left(10^{-5} \mathrm{~g}\right)$ can be obtained. The details of the experiment are given in a later article.

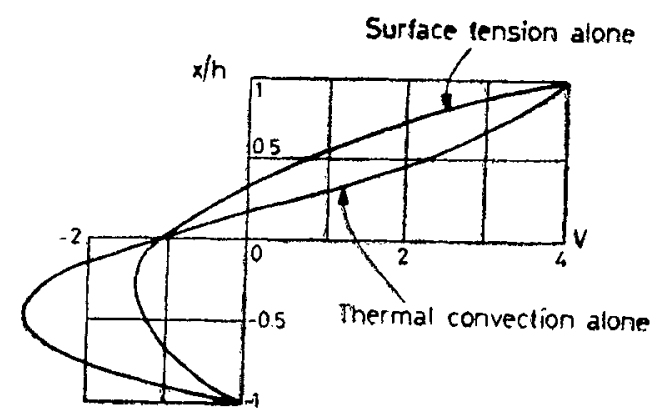

Velocily distributions

Figure 5. Film velocity distributions.

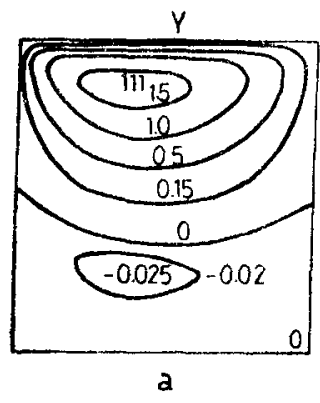

Streamlines

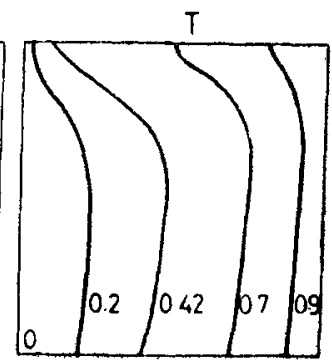

b

Isotherms

Figure 6. Surface-tension flow in a square. 
The experiment clearly indicates that surface tension at reduced gravity can generate significant flows that are somewhat similar to those due to buoyancy at normal gravity.

Unstable Convection - When a fluid layer is heated from below it is found that a cellular flow pattern develops when the temperature gradient exceeds a critical value. This motion is referred to as Benard cells. Such motions can be obtained merely due to the thermal instability previously described. However, when a free surface is present the mation has been shown by Black (1956) to be due to surface tension. The theory of such surface-tension induced motions was further developed by Pearson (1958).

Experiments perfarmed on the flights of Apollo 14 and 17, (Gradzka and Bannister 1972; Bannister 1973; Bannister et al 1973; Grodzka and Bannister 1974) indicated that Benard cells are cbtained in reduced-gravity environments and that, as in a normal gravity environment, a critical temperature gradient must first be exceeded.

Some direct reduced-gravity observations have been obtained for the convection phenomena discussed to date. There are, in addition, other meohanisms possible for generating fluid motions under reduced-gravity conditions. These will now be described but not as comprehensively.

\section{Thermoaconstic convection}

Independent of gravity, thermal volume expansions can act as the driving force for convection. Rapid heating of a confined fluid causes rapid lacal expansions which, in turn, generate pressure waves. These pressure waves produce a convective motion which can greatly increase heat transfer relative to conduction and oan also mass and chemical species transport. This provides a mechanism for enhancing or suppressing convective motion at low gravity by contralling the heating rate. Thermoacoustic convection in a reduced-gravity environment has been theoretically studied by Spradley and Churchill (1975) and Larkin (1967). Significant reduced-gravity thermoacoustic convection in gases is predicted when the heating rates are sufficiently high. For the example treated by Spradley and Churchill (1975) it was found that at normal gravity the gravity-driven convection is so strong as to virtually avershadow the thermoacoustic effects. The two types of convection are of comparable magnitudes at about $10^{-3} \mathrm{~g}_{0}$. At this level the temperature profiles are a bybrid between the buayancy dominated one-g and the thermoacoustic zero- $g$ cases. No experimental evidence of this phenomenon has, as yet, been demonstrated.

\section{Phase-change convection}

A shrinkage usually occurs during solidification because the density of the solid is usually higher than that of the liquid and, hence, accupies a smaller volume. This volume reduction results in a flow of liquid toward the solidifying interface. Such flows are also generated by non-equimolar reactions as occur in vapour deposition orystal growing. Pressure pulses can accompany such flows and, thereby, influence the nature of the material. 
Analyses of this phenomenon are presented by Chambré (1956) and Horvay (1962). Some speculation concerning the influence of phase-change convection on space experiments is presented by Grodzka and Bannister (1974).

\section{Thermosolutal convection}

If a thermal gradient is imposed on a solution its components will migrate so that a concentration gradient will be established. This is called the Sorct effect. Usually the heavier species migrate to the warmer region and the lighter species to the cooler region. If these gradients are normal to the gravitational force (conventional convection) the unusual phenomenon known as salt fingers accurs in which columns of cold fresh water rise and columns of saltier hot water descend (Yih 1970). Other unexpected results are obtained if the gradients are parallel to the gravity vector (unstable convection). The Soret effect has been used for separation processes, but it is relatively incficient in the absence of natural convection because of back diffusion. Laminar convection improves the separation. On earth, however, to maintain laminar convection the flow passagcs have to be quite narrow. In a rcduced-gravity environment, however, laminar convection could be obtained with larger flow passages and, thus, larger samples could be processed. In such a case the low-gravity environment is used to control rather than eliminate convection. Thus, thermosolutal convection at rcduccd-gravity appears to be a promising and beneficial phenomenon.

\section{Convection due to electric and magnetic fields}

Electric and magnetic fields induce bady forces (like gravity) so that they can generate convection and phase separation similar to those in a gravitational field. Whereas in a gravitational field convection is generated by density differences, in an electrical field the flow is generated by differences in electrical conductivity and by differences in susceptibility in a magnetic field. The elcctrical conductivity and magnetic susceptibility are temperature dependent so that tcmperature differences are usually required to obtain the flows. Both conventional and unstable types of convection are possible with such fields. These phenomena have not been fully explored or exploited in the space programme, but may be important if the electromagnetic interferences within spacecraft are not multificd, especially in experiments invalving electrically conducting liquids.

\section{Caupling phenomena}

It should be noted that two or more forces (such as gravity and surface tension) and gradients (such as temperature and concentration) can occur in a given body of fluid. In a sense, thermosolutal phenomena are examples. Their combined action can be such that a given mode of convection is reinforced, annulled, or altcred. What little wark that has been done on problems of this type pertains to very specific situations and the results are not generalizable or predictable. This topic forms the subject of a later article where passible flow configurations are described and related problems are identified. 


\section{Conclusion}

To summarize, various types of reduced-gravity convection possible in a reduced gravity environment are as follows. (a) acceleration-field dependent (i) thermal convection, (ii) solutal convection, (iii) $g$-jitter convection and (iv) thermosolutal convection; (b) independent of acceleration field (i) surface-tension canvection, (ii) thermoacoustic convection, (iii) phase-change convection and (iv) convection due to electric and magnetic fields; (c) coupled or combined convection.

\section{Acknowledgement}

This work was supported under NASA Grant Number NAS 8-31802.

\section{References}

Babskiy V G, Skolvskaya I L and Sklovskiy Y B 1973 Space studies in the Ukraine No 1 Space materials studies and technology (ed.) G S Pisarenko (Kieve : Nankova Domka) p. 121

Bannister T C 1973 Heat flow and convection demonstration Apollo 14 NASA TM X-64735

Bannister T C, Grodzka P G, Spradley L W, Bourgeois S V, Hedden R O and Facemire B R 1973 Apollo 17 heat flow and convection experiments Final data analysis results NASA TM $\mathrm{X}-64772$

Batchelor G K $1954 Q$. Appl. Math. 12

Birikh R V 1966 J. Appl. Mech. Tech. Phys. 369

Black M J 1956 Nature 178650

Catton I and Edwards D K $1967 \mathrm{~J}$. Heat Trans. 89

Catton I 1972 J. Heat Trans. 94

Catton I $1970 \mathrm{~J}$. Heat Trans. 92

Chambre P L 1956 Q.J. Mech. Appl. Math. 9224

Chin J H, Donaldson J O, Gallagher L W, Harper E Y, Hurd S E and Satterlee H M 1969 Analytical and experimental study of liquid orientation and stratification in standard and reduced gravity field LMSC 2-05-64-1 (Palo Alto California : Lockheed Missiles and Space Co.)

Clever R M $1973 \mathrm{~J}$. Heat Trans. 95

Canway B A 1972 Development of Skylab experiment T-013 crew/vehicle disturbance NASA TN D 6584

Davis S H 1967 J. Fluid Mech. 30

Edwards D W and Catton I $1969 \mathrm{~J}$. Heat Mass Trans. 92

Fineblum S S, Haron A S and Saxton J A 1971 Heat Transfer and thermal stratification in the Apollo 14 cryogenic oxygen tanks MSC-04312 MSC Cryogenics symposium papers

Forester C K 1971 Pressurized expulsion of non-isothermal single phase cryogen, MSC cryogenic symposium papers MSC 0431220

Gershuni G Z, Zhukhovitskii E M and Iurkov I S 1970 On convective stability in the presence of a periodically varying parameter; PMM 343470

Grodzka P G 1973 Types of natural convection in space processes summary and report Lockheed Missiles and Space Co Huntsville Research and Engineering Centre HREC-5577-4 CMSC-HREC TR D306350

Grodzka P G and Bannister T C 1972 Science 176506

Grodzka P G and Bannister T C 1974 Natural convection in low-g environments AIAA paper No. 74

Hart J E 1971 J. Fluid Mech. 47

Hart J E 1973 J. Heat Mass Trans. 16

Heitz W L and Westwater J W 1971 J. Heat Trans. 93

Hollands K G T and Konicek L 1973 Int. J. Heat Mass Trans. 16

Horvay G 1962 Proc. US Natl. Cong. Appl. Mech. 2

Jennings P A and Sani R L $1972 J$. Heat Trans. 94 
Larkin B K 1967 Prog. Astronaut. Aeronaut. 20819

Nield D A 1974 J. Fluid Mech. 19341

Ostrach S 1964 Laminar flows with body forces, theory of laminar flows, Vol. 4 high speed aerodynamics and jet propulsion (ed.) F K Moore (Princeton University Press)

Ostrach S 1966 Proc. 3rd Int. Heat Trans. Conf. 6

Ostrach S 1972 Adv. Heat Trans. (Academic Press) 8 Ch. 3

Ostrach S 1976 Proc. 1st Mech. Eng. Cong. (Iran: Pahlavi nverusity)

Ostrach S and Pnueli D 1963 Trans. ASME C85 No. 4

Ozoe H, Sayama H and Churchill S W 1974 Int. J. Heat Mass Tra!'su 17

Ozoe H, Sayama $\mathrm{H}$ and Churchill S W Int. J. Heat Mass Trans. (in press)

Ozoe H, Yamamoto K, Sayama H and Churchill S W 1974 Int. J. Heat Mass Trans. 17

Pak H Y, Wintèr E R F and Schoenals R J 1970 Convection heat transfer in a confined fluid subjected to vibration augmentation of convective heat and mass transfer (eds.) A E Bergles and A L Webb ASME, New York p. 158

Pearson J R A 1958 J. Fluid Mech. 4489

Rice R A 1971 Apollo 14 flight support and systems performance MSC 04312 MSC c1yogeric symposium papers

Richardson P D 1967 Appl. Mech. Rev. 20201

Samuels M R and Churchill S W 1967 AIChE J. 10

Scriven L E and Sternling C V 1974 J. Fluid Mech. 1932

Sherman M and Ostrach S $1967 \mathrm{~J}$. Appl. Mech. E 34 No. 2

Spradley L W, Bourgeois S V and Lin F N 1975 Space processing convection evaluation G-jitter convection of confined fluids in low gravity AIAA paper 75

Spradley L W and Churchill S W 1975 J. Fluid Mech.

Unny T E 1972 J. Appl. Mech. 94

Yih C S 1970 Phys. Fluids 132907 